LA GRANJA:

REVISTA DE

CIENCIAS DE LA VIDA

pISSN:1390-3799; eISSN:1390-8596

http:/ / doi.org/10.17163/lgr.n34.2021.08
Artículo científico / Scientific paper

ANALÍTICA DE CONTAMINANTES

\title{
REVISIÓN SOBRE LIMITES MÁXIMOS DE CADMIO EN CACAO (THEOBROMA CACAO L.)
}

\author{
REVIEW ON MAXIMUM LIMITS OF CADMIUM IN COCOA (Theobroma cacao L.)
}

\author{
Nelino Florida Rofner*[0
}

Carrera profesional de Ingeniería en Conservación de Suelos y Agua. Universidad Nacional Agraria de la Selva-Perú, Av. Universitaria Km 1.5

*Autor para correspondencia: nelinof@hotmail.com

Manuscrito recibido el 30 de octubre de 2020. Aceptado, tras revisión, el 23 de febrero de 2021. Publicado el 1 de septiembre de 2021.

\begin{abstract}
Resumen
El cadmio (Cd) tiende a bioacumularse en granos de Theobroma cacao, afectando la salud humana y sus posibilidades de comercialización. Esto llevó a la Unión Europea (UE) a aprobar el Reglamento $N^{\circ}$ 488/2014 para productos procesados del cacao, y motivó a la comunidad científica a realizar investigaciones sobre su bioacumulación en granos, los potenciales riesgos a la salud, calidad, y sus posibilidades de exportación. Los resultados evidencian altos niveles en diferentes regiones de los principales países productores Latinoamericanos (LA): Brasil, Ecuador, Colombia, Perú, República Dominicana, Bolivia, Honduras, y otros. Sin embargo, el reglamento 488/2014 no estipula límites máximos en cacao sin procesar; en ausencia de este, las investigaciones han clasificado estos límites, tomando como referencia los límites para cacao procesado, generando sobredimensionamiento de los niveles del metal, controversias en el mercado y retroceso en la sustitución del cultivo ilegal de la coca en esta región. Por lo tanto, en este artículo de revisión se detallarán las investigaciones realizadas sobre los niveles de $\mathrm{Cd}$ en granos de cacao en principales países productores de América Latina, la aplicación del reglamento $\mathrm{N}^{\circ} 488 / 2014$ a cacao sin procesar, las propuestas para establecer límites máximos en granos sin procesar y sus implicaciones en la sustitución de cultivos ilícitos.
\end{abstract}

Palabras clave: Cacao sin procesar, cacao de América Latina, cadmio en granos, cultivos ilícitos, límites máximos, reglamento.

\begin{abstract}
Cadmium (Cd) tends to bioaccumulate in Thebroma cacao beans, affecting human health and its marketing possibilities. For this reason, the European Union (EU) approved Regulation No 488/2014 for processed cocoa products, which applies from January 2019, and motivated authors to conduct research on its bioaccumulation in beans, the potential risks to health, quality, and its export possibilities. The results show high levels in different regions of the main Latin
\end{abstract}


American (LA) producing countries: Brazil, Ecuador, Colombia, Peru, the Dominican Republic, Bolivia, Honduras, and others. However, EU regulation does not stipulate maximum limits for raw cocoa. In the absence of it, research has been classified by reference to the limits for processed cocoa, generating oversized metal levels, controversies in the producer's and setback in replacing illegal coca cultivation in this region. Thus, this review article will detail research on $\mathrm{Cd}$ levels in cocoa beans in major Latin American producing countries, the application of EU regulation No $488 / 2014$ to raw cocoa, proposals to set maximum limits on raw beans and their implications for replacing illicit crops.

Keywords: Raw cocoa, Latin American cocoa, beans cadmium, illicit crops, maximum limits, regulations.

Forma sugerida de citar: Florida Rofner, N. (2021). Revisión sobre limites máximos de cadmio en cacao (Theobroma cacao L.). La Granja: Revista de Ciencias de la Vida. Vol. 34(2):117-130. http: / / doi.org/10.17163/lgr.n34.2021.08.

ID Orcid:

Nelino Florida Rofner: http:/ /orcid.org/0000-0002-8751-4367 


\section{Introducción}

El cadmio $(\mathrm{Cd})$ es un metal pesado con propiedades intermedias entre el zinc (Zn) y el mercurio (Hg) (Pérez y Azcona, 2012; Antoine y col., 2017), ampliamente usado en la industria desde hace 50 años (Pérez y Azcona, 2012; Gunnar, 2013). En la actualidad está provocando una serie de transtornos en la salud, con afecciones en órganos vitales: pulmones, riñones, hueso y probablemente desarrollo de carcinogénesis (Reyes y col., 2016), consecuencia de su alta movilidad y poder bioacumulativo (Reyes y col., 2016; Engbersen y col., 2019; Raju y col., 2020; Gunnar, 2013). Los estudios recientes consideran al cadmio junto con el plomo, mercurio y cromo como elementos muy peligrosos para la alimentación humana (Casteblanco, 2018; Engbersen y col., 2019); situación que ha captado la atención de la comunidad científica orientada a su descripción y comportamiento en los sistemas biológicos a plantear alternativas de prevención, control y remediación.

La implementación del Reglamento $\mathrm{N}^{\circ}$ 488/2014 estableció límites tolerables entre 0,1 a $0,8 \mu \mathrm{g} \mathrm{g}^{-1}$ a productos derivados del cacao (Jiménez, 2015; Kruszewski, Wiesław y Kowalska, 2018), mencionados en los reportes científicos previos que mostraban niveles altos de cadmio en suelos (CdS) y granos (CdA) (Prieto y col., 2009; Mite, Carrillo y Durango, 2010; Sánchez, Rivero y Martínez, 2011; Bravo, Arboleda y Martin, 2014) y, propició una carrera científica orientada al tema. Al respecto, las investigaciones han puesto en evidencia que los suelos generalmente tienen bajos niveles de $\mathrm{Cd}$ y su biodisponibilidad depende de las características del suelo (Bravo, Arboleda y Martin, 2014; He y col., 2015; Gramlich y col., 2017; Díaz y col., 2018; Gramlich y col., 2018). También, se reportan concentraciones de CdA superiores al propio suelo, producido por diversos factores entre el sistema suelo-cacao (Chávez y col., 2015; Arévalo y col., 2017b; Gramlich y col., 2017; Hernández y col., 2017; Tantalean y Huauya, 2017; Casteblanco, 2018; Díaz y col., 2018; Florida, Claudio y Gómez, 2018; Gramlich y col., 2018; Kruszewski, Wiesław y Kowalska, 2018; Argüello y col., 2019; Barraza y col., 2019; Florida y col., 2019; Romero y col., 2019; Zug y col., 2019), y en su mayoría reportan valores que superan los límites tolerables establecidos por la UE.
En este contexto, los reportes científicos han tomado como norma de referencia para determinar los niveles de CdA al Reglamento $\mathrm{N}^{\circ}$ 488/2014 (Unión Europea-UE, 2014), que rige desde enero del 2019 (Jiménez, 2015; Kruszewski, Wiesław y Kowalska, 2018; Meter, Atkinson y Laliberte, 2019) y establece límites tolerables entre 0,1 a $0,8 \mu \mathrm{g} \mathrm{g}^{-1}$ a productos derivados del chocolate y no dispone límite máximo para granos sin procesar. Además, existe una clasificación incorrecta al aplicar límites tolerables de productos derivados o procesados a las concentraciones en granos de cacao sin procesar (Pastor, 2017). Por esta razón, debe consignarse o regularse un límite máximo de $\mathrm{Cd}$ en granos secos o masa de cacao sin procesar, utilizando algunos criterios y tomando como base lo ya establecido en el actual reglamento de la UE (Meter, Atkinson y Laliberte, 2019). En este contexto, el objetivo de este trabajo de revisión es detallar las investigaciones realizadas sobre los niveles de Cd en cacao de los principales productores en América Latina; analizar la aplicación del actual reglamento de la UE; exponer las propuestas de límites máximos en granos sin procesar y las implicancias del vacío normativo en el productor y en la sustitución de cultivos ilícitos en la región.

\section{Metodología}

Se realizó una búsqueda en las bases de datos de Scielo, Web of Science y Scopus, enfocándose en el tema del cadmio realizados en los principales países productores de cacao en América Latina como Perú, Colombia, Ecuador, Brasil, Bolivia, Honduras, Venezuela y Republica Dominicana. La búsqueda se realizó restringiendo los resultados con las palabras clave: cadmio, cadmio en cacao, cadmio en plantas, toxicidad del cadmio y cadmio en la salud.

Se identificó que el tema es tratado principalmente en las revistas: Science of The Total Environment, Water Air Soil Pollution, Ecotoxicology and Environmental Safety, Acta Agronómica y otros como fuentes primarias, y como fuentes secundarias a instituciones como Organización de las Naciones Unidas para la Alimentación y la Agricultura (FAO), Unión Europea (UE), Codex Alimentarius (CODEX), Agencia de Protección Ambiental de Estados Unidos (USEPA) y los sectores gubernamentales del Perú: Ministerio de Agricultura y Riego - 
MINAGRI y el Instituto de Estadística e Informática - INEI. Posteriormente, se amplió la búsqueda a otras revistas a través de buscadores como Google Scholar, así como congresos organizados por la International Cocoa Organization (ICO). Se seleccionaron las investigaciones de los últimos 10 años, salvo casos particulares de artículos que son citados con mucha frecuencia en el resto de las publicaciones analizadas en esta revisión.

\section{Cd en cacao de origen Latino Americano}

Antes del 2014 y entre el 2014 al 2019, periodo de adaptación a la entrada en vigencia del reglamento de la UE y posterior a la entrada en vigencia, se han realizados una serie de trabajos (Tabla 1) en los principales productores en LA, a escalas nacionales, regionales y focalizadas en áreas experimentales.

La Tabla 1 muestra los reportes científicos publicados en revistas indexadas y arbitradas, y los países con más reportes científicos son Ecuador y Perú; además, los valores medios más altos reportados lo encabezan Perú, Costa Rica y Venezuela. La clasificación de los niveles de Cd fue realizada según los límites tolerables del Reglamento $\mathrm{N}^{\circ} 488 / 2014$ de la UE, que fija un límite tolerable máximo de $0,8 \mu \mathrm{g}$ $\mathrm{g}^{-1}$ a chocolates con sólidos de cacao superiores o iguales al 50\%; según este criterio, el 70,59\% de los reportes corresponden a niveles altos de cadmio y solo $29,4 \%$ estarían dentro de los limites exigidos por la UE.

Los resultados mostrados (Tabla 1), independientemente del método de su determinación, fueron clasificados en algunos casos tomando como referencia y en otros como norma de estricta aplicabilidad al Reglamento de la UE, a pesar de que se trata de niveles de $\mathrm{Cd}$ en granos sin procesar. $\mathrm{Al}$ respecto, se muestran concepciones distintas respecto a la aplicación del Reglamento de la UE:

1. Autores que diferencian la aplicabilidad del reglamento de la UE, entre ellos Barraza y col. (2017) y Furcal y Torres (2020) explican que la Autoridad Europea de Seguridad Alimentaria no ha establecido un límite para el $\mathrm{Cd}$ en la materia prima del chocolate, y que los estudios demuestran que las concentraciones de
$\mathrm{Cd}$ en granos pueden alcanzar niveles superiores a lo establecido por la UE.

2. Autores que diferencian, pero consideran indistinta su aplicabilidad, entre ellos Lanza y col. (2016) señalan que el límite máximo de la UE podría aplicar de igual manera para el grano de cacao y que sus contenidos hallados de $C d\left(1,62 \mu \mathrm{g} \mathrm{g}^{-1}\right)$ superan el valor máximo establecido en el Reglamento de la UE.

3. Autores que no diferencian la aplicabilidad del reglamento de la UE, entre ellos Gramlich y col. (2018), señalan que las concentraciones de $C d$ en granos $\left(1,1 \mu \mathrm{g} \mathrm{g}^{-1}\right)$ exceden el límite propuesto por la UE. Con este mismo criterio, Arévalo y col. (2017a) clasifican como altos los niveles de $\mathrm{Cd}\left(1,13 \mu \mathrm{g} \mathrm{g}^{-1}\right)$ en granos de cacao cultivado en las regiones de Amazonas, Piura y Tumbes (Zona norte del Perú) en alusión a la norma de la UE. Florida, Claudio y Gómez (2018) clasifican sus niveles de Cd $\left(0.98 \mu \mathrm{g} \mathrm{g}^{-1}\right)$, como mayor al permitido por la UE. También, Zug y col. (2019) señalan el Cd en semillas de cacao peruano como el más alto con los límites permitidos, en particular el CCN-51 en comparación al cacao fino y de sabor. Finalmente, Chávez y col. (2015) no solo aplican incorrectamente el Reglamento, también se equivocan al señalar que el $\mathrm{Cd}$ en granos por encima de un nivel crítico $0,6 \mu \mathrm{g} \mathrm{g}^{-1}$ causaría preocupación en el consumo de chocolate a base de cacao, cuando el Reglamento tolera hasta $0,8 \mu \mathrm{g} \mathrm{g}^{-1}$.

\section{Reglamento 488/2014 UE}

El actual reglamento de la UE a cargo de la Autoridad Europea de Seguridad Alimentaria (EFSA) fue elaborado por la Comisión Técnica de Contaminantes de la Cadena Alimentaria (CONTAM). La EFSA consideró necesario modificar nuevamente los niveles máximos para ciertos contaminantes como el Cd, consignados en el Reglamento 1881/2006, incorporando nueva información y los avances del Codex Alimentarius (Unión Europea-UE, 2014; Zug y col., 2019; Furcal y Torres, 2020).

El Reglamento actual de la UE se basa en tres aspectos fundamentales: 
1. Exposición alimentaria; la CONTAM realizó los estudios de ingesta semanal tolerable y determinó la exposición alimentaria media de $\mathrm{Cd}$ en los países europeos de $2,5 \mu \mathrm{g} / \mathrm{kg}$ de peso corporal (Unión Europea-UE, 2014; Abt y Lauren, 2020).

2. El consumo percápita; un elevado consumo de derivados del cacao puede elevar los niveles de cadmio en el cuerpo (Tabla 2); y en el caso de la comunidad europea los consumos percápita triplican los consumos de países Latinoamericanos.

3. Principio de ALARA, por sus siglas en ingles "As Low As Reasonably Achievable" que significa tan bajo como sea razonablemente alcanzable o posible (Unión Europea-UE, 2014).

Para la EFSA es razonable que la reducción a la exposición de los consumidores vulnerables podría lograrse mediante el establecimiento de un contenido máximo a los derivados del cacao. Así, el 12 de mayo del 2014 fue aprobado el Reglamento $488 / 2014$ que modifica el Reglamento 1881/2006 (Tabla 3), donde se añade a la lista de productos controlados los derivados del cacao (Unión EuropeaUE, 2014; Gramlich y col., 2018; Kruszewski, Wiesław y Kowalska, 2018; Argüello y col., 2019; Barraza y col., 2019; Romero y col., 2019; Zug y col., 2019; Abt y Lauren, 2020).

Este Reglamento asigna un alto valor a chocolates con porcentaje total de materia seca $\geq 50 \%$ (Antolinez y col., 2020), establece límites tolerables para $\mathrm{Cd}$ en cuatro tipos de chocolate (consumo final), pero utiliza argumentos de los Estándares de Calidad Ambiental (ECA) y es poco coherente al fijar valores similares a alimentos muy diferentes en origen y representatividad en la exposición alimentaria total de cadmio de los consumidores. De igual forma, tiene valores con exceso y sin base científica suficiente que pueden convertirse en trabas para el proceso productivo y representar obstáculos técnicos al comercio, al confundir los límites tolerables en los productos derivados o procesados para la comercialización del cacao en grano (Pastor, 2017).

\section{Propuestas de límites máximos para cacao sin procesar}

La categorización de los niveles de cadmio total en granos sin procesar utilizando el Reglamento 488/2014 es un error (Pastor, 2017; Meter, Atkinson y Laliberte, 2019), pues la precitada norma de la UE (Tabla 3) no es aplicable a granos enteros sin procesar, aunque, como ya se explicó, la mayoría de los autores señalan que sus valores encontrados superan lo establecido por la UE que fija un máximo de $0,8 \mu \mathrm{g} \mathrm{g}^{-1}$, por lo que tácitamente se entiende que se está usando este límite para clasificar sus niveles encontrados. Debe tomarse en cuenta que la inmensa mayoría del cacao se exporta desde Perú y otros países de la región en forma de grano fermentado seco (MINAGRI, 2019). Paradójicamente, los límites tolerables para chocolate están siendo utilizados para juzgar y ajustar el precio del grano sin procesar (Pastor, 2017). Por tanto, a continuación se analizan algunas propuestas que no alteran los criterios que originaron el actual Reglamento e incorporan nuevos criterios como una simple relación de proporcionalidad (Meter, Atkinson y Laliberte, 2019).

\section{a) Propuesta de Meter}

Una de las propuestas para niveles de Cd en granos ha sido establecida por Meter, Atkinson y Laliberte (2019), quienes aplican una relación de proporcionalidad a los límites fijados en el Reglamento de la UE y calculan un valor límite máximo de Cd en granos secos sin procesar, ya que la masa sin procesar contiene una cantidad similar de $\mathrm{Cd}$ al de los granos de origen.

Esta propuesta asume los siguientes conceptos:

- La regulación 488/2014 es para productos procesados.

- La concentración de Cd en masa es similar al licor de cacao (primer derivado del procesamiento).

- Se conoce el\% de masa en el chocolate

- La manteca contiene niveles mínimos de Cd (criterio no aplicado en su fórmula)

- Proporcionalidad 
La fórmula de cálculo es:

$$
M L C M=\frac{M L E U \cdot P}{X \% P}
$$

Donde:

$M L C M=$ Nivel máximo de Cd en la masa del cacao $\left(\mu \mathrm{g} \mathrm{g}^{-1}\right)$

$M L E U \cdot P=$ Nivel máximo de la UE en producto final $\mathrm{P}\left(\mu \mathrm{g} \mathrm{g}^{-1}\right)$

$X \% P=$ Porcentaje de masa en producto terminado $\mathrm{P}$

Pone como ejemplo el chocolate oscuro con $70 \%$ de masa (sólidos secos de cacao), y la UE establece $0,8 \mu \mathrm{g} \mathrm{g}^{-1}$ de $\mathrm{Cd}$ en el producto terminado, por lo que el nivel máximo de $\mathrm{Cd}$ será:

$$
M L C M=\frac{0,8}{0,7}=1,14 \mu \mathrm{g} \mathrm{g}^{-1}
$$

Se puede observar que los niveles máximos de la UE son para los productos terminados y no para la materia prima. La ecuación estima un nivel máximo de Cd en la masa de $1,14 \mu \mathrm{g} \mathrm{g}^{-1}$ que garantizará que el producto final permanezca por debajo del umbral fijado por la UE.

\begin{tabular}{|c|c|c|c|c|c|}
\hline Referencias & País & $\begin{array}{l}\text { Media de Cd } \\
\quad\left(\mu \mathrm{g} \mathrm{g}^{-1}\right)\end{array}$ & Min. - Max. & $\begin{array}{c}\text { Método } \\
\text { determinación }\end{array}$ & $\begin{array}{c}\text { Nivel } \\
\text { reportado, } \\
\text { según } \\
\text { Reglamento } \\
\mathrm{N}^{\circ} 488 / 2014\end{array}$ \\
\hline \multirow{2}{*}{ Furcal y Torres (2020) } & Costa & $0,44 \pm 0,64^{*}$ & $0,0-1,8$ & \multirow{2}{*}{ ICP OES } & $\mathrm{Nb}$ \\
\hline & Rica & $2,25 \pm 2,06^{* * *}$ & $0,0-8,7$ & & $\mathrm{Na}$ \\
\hline Argüello y col. (2019) & \multirow{6}{*}{ Ecuador } & $0,9 \pm-$ & - & ICP MS & $\mathrm{Na}$ \\
\hline Barraza y col. (2019) & & $1,26 \pm 0,18$ & - & ICP-MS & $\mathrm{Na}$ \\
\hline Barraza y col. (2017) & & $1,12 \pm-$ & - & ICP-MS & $\mathrm{Na}$ \\
\hline Chávez y col. (2015) & & $0,94 \pm-$ & - & EAA & $\mathrm{Na}$ \\
\hline Mite, Carrillo y Durango (2010) & & $0,84 \pm 0,32$ & $0,32-1,80$ & EAA & $\mathrm{Na}$ \\
\hline Romero y col. (2019) & & $0,75 \pm-$ & - & ICP OES & $\mathrm{Nb}$ \\
\hline Lanza y col. (2016) & Venezuela & $1,62 \pm-$ & $0,95-2,09$ & ICP OES & $\mathrm{Na}$ \\
\hline Oliveira y col. (2019) & Brasil & $0,13 \pm-$ & $0,04-0,82$ & ICP OES & $\mathrm{Nb}$ \\
\hline \multirow[t]{2}{*}{ Gramlich y col. (2018) } & Honduras & $1,10 \pm 0,2$ & - & \multirow{4}{*}{ ICP OES } & $\mathrm{Na}$ \\
\hline & \multirow{6}{*}{ Perú } & $1,13^{*} \pm-$ & - & & $\mathrm{Na}$ \\
\hline Arévalo y col. (2017a) & & $0,45^{* *} \pm-$ & - & & $\mathrm{Nb}$ \\
\hline & & $0,20^{* * *} \pm-$ & - & & $\mathrm{Nb}$ \\
\hline Florida, Claudio y Gómez (2018) & & $0,98 \pm 1,42$ & $0,18-6,7$ & EAA & $\mathrm{Na}$ \\
\hline Tantalean y Huauya (2017) & & $1,08 \pm-$ & - & EAA & $\mathrm{Na}$ \\
\hline Zug y col. (2019) & & $2,46 \pm 0,75$ & $0,2-12,56$ & GFAAS & $\mathrm{Na}$ \\
\hline \multicolumn{5}{|c|}{ Resultados que superan los límites según este criterio (\%) } & 70,59 \\
\hline
\end{tabular}

Tabla 1. Cd en granos de cacao (Theobroma cacao L.) en países Latinoamericanos

${ }^{*}$ zona norte, ${ }^{* *}$ zona central, ${ }^{* * *}$ zona sur del país correspondiente, $\mathrm{Na}$ nivel alto, $\mathrm{Nb}$ nivel bajo, EAA espectrofotómetro de absorción atómica, ICP OES espectrometría de emisión óptica con acoplamiento inductivo, ICP-MS Espectroscopia de masas de plasma con acoplamiento inductivo, GFAAS espectrómetro de absorción atómica de horno de grafito, - no especificado por el autor.

\section{b) Propuesta del autor}

La propuesta toma como base los cálculos de Meter, Atkinson y Laliberte (2019), las conclusiones de Pastor y Gutierrez (2016) y Pastor (2017) y los conceptos generales de la composición bromatológica media del chocolate y de los granos sin proce- sar (Tabla 4); por lo tanto, la propuesta asume los siguientes conceptos:

- Químicamente el cacao está constituido por: Grasa o manteca de cacao 53,05\% usado para chocolate y la diferencia es torta de cacao utilizado para cacao en polvo edulcorado pa- 
ra beber (Morales, García y Méndez, 2012), y toma el factor de 0,5 (\% TA).

- Los chocolates amargos poseen manteca de cacao y en promedio no superan el 50\% (Sánchez y col., 2016).

- En el chocolate al 70\% de manteca de cacao, el contenido de $\mathrm{Cd}$ se reduce a menos de la mitad en el chocolate en comparación al grano, por ello, se aplica un factor de 0,5 (RP) (Pastor y Gutierrez, 2016).
- La manteca contiene niveles mínimos de Cd (Meter, Atkinson y Laliberte, 2019), aspecto que no fue considerado en su fórmula y confirma lo señalado por Pastor y Gutierrez (2016).

- Los granos bioacumulan cadmio en concentraciones variadas según el genotipo de cacao (Tabla 5), con una variación aproximada de $30 \%$, proporción que debe eliminarse aplicando un factor de 0,7 (VG) a los resultados parciales de manteca y torta de cacao.

Tabla 2. Consumo percápita de chocolate en Europa y América Latina

\begin{tabular}{ccccc}
\hline Continente & País & $\begin{array}{c}\text { Consumo } \\
\text { anual por } \\
\text { persona } \\
(\mathrm{kg})\end{array}$ & $\begin{array}{c}\text { Barras } \\
\text { anuales } \\
\text { (barra / 70g) }\end{array}$ & Barras/mes \\
\hline \multirow{6}{*}{ Europa } & Suiza & 11,9 & 170 & 14 \\
& Irlanda & 9,9 & 141 & 12 \\
& Reino Unido & 9,5 & 136 & 11 \\
& Austria & 8,8 & 126 & 10 \\
& Bélgica & 8,3 & 119 & 10 \\
& Alemania & 8,2 & 117 & 10 \\
\hline Media & $9,43 \pm 1,38$ & $134,8 \pm 19,61$ & $11,17 \pm 1,6$ \\
\hline & Uruguay & 3,1 & 44 & 3,7 \\
& Argentina & 2,9 & 41 & 3,5 \\
& Chile & 2,2 & 31 & 2,6 \\
& Brasil & 1,7 & 24 & 2 \\
& México & 0,7 & 10 & 0,8 \\
& Perú & 0,6 & 9 & 0,7 \\
\hline & Media & $1,87 \pm 1,07$ & $26,5 \pm 15$ & $2,22 \pm 1,3$ \\
\hline
\end{tabular}

Fuente: Jiménez (2015)

Tabla 3. Límites tolerables Reglamento 488/2014

\begin{tabular}{lcc}
\hline \multicolumn{1}{c}{ Producto } & $\begin{array}{c}\text { Materia seca } \\
(\%)\end{array}$ & $\begin{array}{c}\text { Límite máximo } \\
\text { Permitido } \\
\left(\mu \mathrm{g} \mathrm{g}^{-1}\right)^{*}\end{array}$ \\
\hline Chocolate de leche & $<30$ & 0,1 \\
Chocolate de leche & $\geq 30$ & 0,3 \\
Chocolate & $\geq 50$ & 0,3 \\
Chocolate & 0,8 \\
Cacao en polvo vendido al consumidor final o & \\
como ingrediente en cacao en polvo edulcorado & 0,6 \\
vendido al consumidor final (chocolate para beber) & \\
\hline * Entraron en vigencia desde enero del 2019 & \\
Fuente: Unión Europea-UE (2014), Zug y col. (2019) y Abt y Lauren (2020)
\end{tabular}


Según la Tabla 5, los productos del procesamiento primario de los granos son aproximadamente $50 \%$ de torta de cacao usado para chocolate en polvo edulcorado para beber y con un límite tolerable según la UE de $0,6 \mu \mathrm{g} \mathrm{g}^{-1}$ y manteca de cacao en proporciones similares al $50 \%$, usados para chocolates con un nivel máximo de $0,8 \mu \mathrm{g} \mathrm{g}^{-1}$ (Sánchez y col., 2016). La propuesta sugiere que deben calcularse por separado la manteca (Fórmula 4) y la torta (Fórmula 5), y en ambos casos se debe incorporar la variación por genotipo (Tabla 5), para reducir en un $30 \%$ este resultado parcial, aplicando un factor de 0,7; finalmente debe promediarse para obtener un límite máximo (Fórmula 3). Por lo que la fórmula para el cálculo es:

$$
\begin{gathered}
L C P=\frac{L C M C+L C T C}{2} \\
L C M C=\frac{F M}{R P} \times V G \\
L C T C=\frac{M C P}{\% T A} \times V G
\end{gathered}
$$

Donde:

$L C P=$ Limite de $\mathrm{Cd}$ propuesto

$L C M C=$ Límite de Cd en manteca de cacao

$L C T C=$ Limite de Cd en torta de cacao (chocolate en polvo)

$F M=$ Formula de Meter, Atkinson y Laliberte (2019), obtiene un límite máximo de 1,14 $\mu \mathrm{g} \mathrm{g}^{-1}$

$R P=$ Reducción de Cd reportado por Pastor y Gutierrez (2016)

$V G=$ Variabilidad por genotipo, se eliminó el 30\% (aplicando un factor 0,7 al contenido de Cd en manteca y torta de cacao)

$M C P=$ Máximo para cacao en polvo según UE $(0,6$ $\left.\mu \mathrm{g} \mathrm{g}^{-1}\right)$

$\% T A=\%$ de torta en granos sin procesar $50 \%$ (factor 0,5$)$

Como ejemplo, se utiliza el chocolate oscuro con $70 \%$ de masa de cacao $\left(0,8 \mu \mathrm{g} \mathrm{g}^{-1}\right)$, usado y calculado en la formula Meter, Atkinson y Laliberte (2019):

$$
\begin{aligned}
& L C M C=\frac{1,14}{0,5} \times 0,7=1,6 \mu \mathrm{g} \mathrm{g}^{-1} \\
& L C T C=\frac{0,6}{0,5} \times 0,7=0,84 \mu \mathrm{g} \mathrm{g}^{-1} \\
& L C P=\frac{1,6+0,84}{2}=1,22 \mu \mathrm{g} \mathrm{g}^{-1}
\end{aligned}
$$

La ecuación propuesta estima un nivel máximo de Cd en granos sin procesar de 1,22 $\mu \mathrm{g} \mathrm{g}^{-1}$ (Formula 8) garantizando que el producto final procesado esté por debajo del límite fijado por la UE $(0,8 \mu \mathrm{g}$ $\left.\mathrm{g}^{-1}\right)$. Por tanto, de acuerdo con la propuesta de Meter, Atkinson y Laliberte (2019) la categorización de los reportes científicos (Tabla 6) varían razonablemente.

La Tabla 6 muestra que la propuesta del autor supera ligeramente a Meter, Atkinson y Laliberte (2019) y es similar a lo establecido en Indonesia, que establece límites máximos de $1 \mu \mathrm{g} \mathrm{g}^{-1}$ para masa de cacao. Además, el\% de resultados que superarían los límites son similares al comparar los reportes disponibles, sin embargo, en comparación con el Reglamento 488/2014, los niveles categorizados como altos, según los límites máximos propuestos, disminuyen de 70,59 a 23,53\%, cuya cifra es más razonable y coherente. Además, en este análisis aún no están incorporando conceptos de biodisponibilidad del metal, ya que las investigaciones revelan una gran dinámica en concentración y disponibilidad en suelos (Prieto y col., 2009; Kabata, 2010; Sánchez, Rivero y Martínez, 2011; Bravo, Arboleda y Martin, 2014; Gramlich y col., 2017; Díaz y col., 2018; Gramlich y col., 2018; Zug y col., 2019), que pueden contribuir o limitar la movilización y captación del cadmio por el cacao. Por tanto, es necesario que se tomen las acciones correspondientes para subsanar el vacío normativo en protección de la salud y de los millones de productores de cacao en LA y el mundo.

\section{Implicancia del vacío normativo en el productor y la sustitución de cultivos ilícitos}

En la última década el cacao fue el segundo cultivo alternativo a la sustitución de la hoja de coca en el Perú (INEI, 2017). El cacao es el cultivo más exitoso en la sustitución del cultivo ilegal de la coca, y miles de pequeños agricultores familiares han sido rescatados del sembrado de la coca gracias al promisorio cultivo del cacao (Pastor y Gutierrez, 2016). Solo en Perú se cultiva en 16 regiones, 57 provincias y 259 distritos (MINAGRI, 2019) y de igual forma Colombia, Bolivia y Ecuador muestran un crecimiento sostenido en la producción de cacao y una reduc- 
ción de cultivos ilícitos (Celis, Florida y Rengifo, 2020).

De manera general, en estos países de la región la sostenibilidad de la producción de cacao se encuentra amenazada (Argüello y col., 2019; Abt y Lauren, 2020) y se pueden identificar algunos aspectos influyentes, entre ellos:

1. Reglamento 488/2014; La imposición normativa de la UE de un límite máximo muy alto a los derivados del cacao, considerando que más del $60 \%$ del volumen de producción de América Latina se exportan hacia la UE (MINAGRI, 2016; Meter, Atkinson y Laliberte, 2019).

2. Erradicación forzada; países como Perú, a través de la Comisión Nacional para el Desarro- llo y Vida sin Drogas - DEVIDA y la Agencia de los Estados Unidos para el Desarrollo Internacional- USAID, realizan la erradicación forzada, eliminando en su totalidad el cultivo ilícito de la hoja de coca, trayendo mayores desequilibrios socioeconómicos (Chocce, 2015).

3. Ineficiencia de la inversión pública; causado por factores individuales e institucionales. Los proyectos alternativos (cacao) al cultivo ilícito de la hoja de coca y los proyectos de desarrollo rural no generan mejoras socioeconómicas que el productor espera; esto se evidenció en Perú (Alvarado, Ferrer y Florida, 2020) y en Ecuador (Viteri, 2013) y es muy probable que ocurra lo mismo en los demás países productores de la región.

Tabla 4. Caracterización fisicoquímica en granos de cacao (Theobroma cacao L.)

\begin{tabular}{lcc}
\hline \multicolumn{1}{c}{ Parámetros } & Origen del cultivo & $\begin{array}{c}\text { Contenido } \\
\text { medio }\end{array}$ \\
\hline Cascarilla\% & Físicos \\
Peso del grano (g) & & $11-12$ \\
Humedad (\%) & & $1,05-1,2$ \\
\hline & Químicos & $7-8$ \\
\hline & Cacao de montaña \\
Grasa (\%) & Bosque húmedo tropical & 55 \\
& Valles interandino seco & 54 \\
Proteína (\%) & Cacao de montaña & 14 \\
& Bosque húmedo tropical \\
pH & Valles interandino seco & 13 \\
& Cacao de montaña & 13 \\
& Bosque húmedo tropical & 5,07 \\
& Valles interandino seco & 5,97 \\
Fibra (\%) & Cacao de montaña & 3 \\
& Bosque húmedo tropical & 3 \\
Calorías & Valles interandino seco & 3 \\
(kcal/100 g) & Cacao de montaña & 629 \\
& Bosque húmedo tropical & 629 \\
& Valles interandino seco & 625 \\
\hline
\end{tabular}

* Calculado a partir del estudio de 15 genotipos de cacao Fuente: Ministerio de Agricultura y Desarrollo Rural - MINAGRICULTURA (2004) 
Lo delicado de esto es que tanto la erradicación forzada como la ineficiencia en la inversión pública son problemas con las que nuestra región ha convivido por décadas y la aparición y vigencia del Reglamento 488/2014 juega un rol desencadenante a los anteriores, en este caso desfavorable para el productor, y puede desalentar a las familias que han sustituido el cultivo de la coca por el cacao (Pastor, 2017; Abt y Lauren, 2020). Por lo tanto, los niveles tolerables de $\mathrm{Cd}$ en cacao procesado fijado por la UE y aplicados para clasificar los niveles en granos sin procesar es un error (Pastor, 2017; Meter, Atkinson y Laliberte, 2019) y requiere que los organismos como el Codex y la UE considere evaluar, recalcular y consignar límites tolerables de $\mathrm{Cd}$ en granos de cacao sin procesar, para evitar el sobredimensionamiento de la problemática que representa hoy la presencia de este metal en el cacao de América Latina.

Para contextualizar las implicancias, hasta el 2016 en LA y el Caribe, el cultivo se ha difundido a nivel comercial en 23 países, con un volumen superior a las $675000 \mathrm{t}$ y alrededor de $1700000 \mathrm{ha}$, donde Brasil, Ecuador, República Dominicana, Perú, Colombia y México representan más del 90\% de la producción (Arvelo y col., 2017) y al 2018 LA aporta con más del $18 \%$ de la importación mundial de cacao en grano (836 000 t en el período 2017 2018) y en casos como el Perú, del total exportado en 2018, el 54,3\% corresponde a cacao en granos sin procesar (López, Cunias y Carrasco, 2020). Además, países como Ecuador, Perú y Colombia han mostrado un crecimiento medio mayor a $9 \%$ anual en la última década (MINAGRI, 2016; Meter, Atkinson y Laliberte, 2019), y en Ecuador pasó de 3 a 6\% de la producción mundial, llegando a ocupar el cuarto lugar entre los países productores, superando a Brasil (Cunha, 2018).

Finalmente, la falta de límites máximos al cacao sin procesar es advertida como amenaza a la sostenibilidad de la producción de cacao (Argüello y col., 2019; Abt y Lauren, 2020) y viene causando algunas confusiones y especulaciones, entre ellas: a) confusión en la comunidad científica al clasificar cacao sin procesar, aplicando los límites de la norma europea para cacao procesados; $b$ ) sobredimensionada preocupación al sector cacaotero en toda nuestra región de América Latina y $c$ ) distorsiones de mercado al momento de negociar, pues el productor difícilmente está en condiciones de rebatir y los compradores prefieren contenidos bajos de $\mathrm{Cd}$ para garantizar su utilización en cualquier receta, con el consiguiente efecto negativo en el precio que recibe por el grano (Pastor, 2017; Meter, Atkinson y Laliberte, 2019).

Tabla 5. Niveles de Cd en diferentes genotipos de cacao (Theobroma cacao L.)

\begin{tabular}{llc}
\hline \multicolumn{1}{c}{ Referencia } & \multicolumn{1}{c}{ Genotipo } & $\begin{array}{c}\text { Cd granos } \\
\left(\mu \mathrm{g} \mathrm{g}^{-1}\right)\end{array}$ \\
\hline Barraza y col. (2017) & CCN-51 & 1,21 \\
& Nacional Ecuatoriano & 0,89 \\
& HNF & 2,09 \\
& PNF & 1,9 \\
& PF & 1,82 \\
Lanza y col. (2016) & PFC & 1,76 \\
& HF & 1,74 \\
& PFM & 1,57 \\
& HFC & 1,1 \\
Florida, Claudio y Gómez (2018) & HFM & 0,95 \\
\hline Concentración media & & 0,98 \\
CV (\%) & & $1,45+0,43$ \\
\hline
\end{tabular}

CV Coeficiente de variación 
Tabla 6. Comparación de la clasificación de niveles de cadmio

\begin{tabular}{|c|c|c|c|c|c|}
\hline Referencias & País & $\begin{array}{l}\text { Media de } \\
\qquad \mathrm{Cd} \\
\left(\mu \mathrm{g} \mathrm{g}^{-1}\right)\end{array}$ & $\begin{array}{l}\text { Nivel según } \\
\text { Reglamento } \\
\mathrm{N}^{\circ} 488 / 2014\end{array}$ & $\begin{array}{c}\text { Nivel } \\
\text { según } \\
\text { Meter, Atkinson y Laliberte }(2019)^{\mathrm{a}}\end{array}$ & $\begin{array}{l}\text { Nivel según } \\
\text { propuesta } \\
\text { del Autor }\end{array}$ \\
\hline \multirow{2}{*}{ Furcal y Torres (2020) } & Costa & $0,44^{*}$ & $\mathrm{Nb}$ & $\mathrm{Nb}$ & $\mathrm{Nb}$ \\
\hline & Rica & $2,25^{* * *}$ & $\mathrm{Na}$ & $\mathrm{Na}$ & $\mathrm{Na}$ \\
\hline Argüello y col. (2019) & \multirow{6}{*}{ Ecuador } & 0,9 & $\mathrm{Na}$ & $\mathrm{Nb}$ & $\mathrm{Nb}$ \\
\hline Barraza y col. (2019) & & 1,26 & $\mathrm{Na}$ & $\mathrm{Na}$ & $\mathrm{Na}$ \\
\hline Barraza y col. (2017) & & 1,12 & $\mathrm{Na}$ & $\mathrm{Nb}$ & $\mathrm{Nb}$ \\
\hline Chávez y col. (2015) & & 0,94 & $\mathrm{Na}$ & $\mathrm{Nb}$ & $\mathrm{Nb}$ \\
\hline Mite, Carrillo y Durango (2010) & & 0,84 & $\mathrm{Na}$ & $\mathrm{Nb}$ & $\mathrm{Nb}$ \\
\hline Romero y col. (2019) & & 0,75 & $\mathrm{Nb}$ & $\mathrm{Nb}$ & $\mathrm{Nb}$ \\
\hline Lanza y col. (2016) & Venezuela & 1,62 & $\mathrm{Na}$ & $\mathrm{Na}$ & $\mathrm{Na}$ \\
\hline Oliveira y col. (2019) & Brasil & 0,13 & $\mathrm{Nb}$ & $\mathrm{Nb}$ & $\mathrm{Nb}$ \\
\hline \multirow[t]{2}{*}{ Gramlich y col. (2018) } & \multirow[t]{2}{*}{ Honduras } & 1,1 & $\mathrm{Na}$ & $\mathrm{Nb}$ & $\mathrm{Nb}$ \\
\hline & & $1,13^{*}$ & $\mathrm{Na}$ & $\mathrm{Nb}$ & $\mathrm{Nb}$ \\
\hline \multirow[t]{2}{*}{ Arévalo y col. (2017a) } & \multirow{5}{*}{ Perú } & $0,45^{* *}$ & $\mathrm{Nb}$ & $\mathrm{Nb}$ & $\mathrm{Nb}$ \\
\hline & & $0,20^{* * *}$ & $\mathrm{Nb}$ & $\mathrm{Nb}$ & $\mathrm{Nb}$ \\
\hline Florida, Claudio y Gómez (2018) & & 0,98 & $\mathrm{Na}$ & $\mathrm{Nb}$ & $\mathrm{Nb}$ \\
\hline Tantalean y Huauya (2017) & & 1,08 & $\mathrm{Na}$ & $\mathrm{Nb}$ & $\mathrm{Nb}$ \\
\hline Zug y col. (2019) & & 2,46 & $\mathrm{Na}$ & $\mathrm{Na}$ & $\mathrm{Na}$ \\
\hline \multicolumn{3}{|c|}{ Resultados que superan los límites según criterios (\%) } & 70,59 & 23,53 & 23,53 \\
\hline
\end{tabular}

${ }^{\mathrm{a}}$ Max: $1.14 \mu \mathrm{g} \mathrm{g}^{-1}$, b Max: $1.22 \mu \mathrm{g} \mathrm{g}^{-1}$, Na: nivel alto, $\mathrm{Nb}$ : nivel bajo

\section{Conclusiones}

Los reportes científicos revelan avances importantes sobre el $\mathrm{Cd}$ en granos de cacao. En la región, los valores medios más altos reportados lo encabezan Perú, Costa Rica, Venezuela y Ecuador. Además, el Reglamento $N^{\circ}$ 488/2014 de la Unión Europea establece límites máximos para cacao procesados, y está siendo utilizado como base para clasificar la concentración de cadmio en granos sin procesar, generando confusión en la comunidad científica y sobredimensionada preocupación al sector cacaotero.

Existen propuestas de cálculo para determinar un límite máximo razonable y científico, incorporando criterios de proporcionalidad, variabilidad genética y aspectos bromatológicos del cacao, tomando como base los límites ya establecidos en el reglamento de la Unión Europea. Las propuestas de cálculo determinan un límite máximo de cadmio en granos sin procesar de 1,14 (Meter) y 1,22 $\mu \mathrm{g} \mathrm{g}^{-1}$ (autor), reduciendo los niveles categorizados como altos (según la UE), de 70,59 a solo $23,53 \%$, cifra razonable y coherente que contribuirá a mantener la calidad del producto para el consumidor final, evitar distorsiones del mercado protegiendo al produc- tor y a los esfuerzos de sustitución del cultivo ilegal de la coca en Perú, Colombia, Bolivia y otros países de la región.

\section{Referencias}

Abt, E. y P. Lauren (2020). «Perspective on Cadmium and Lead in Cocoa and Chocolate». En: Journal of Agricultural and Food Chemistry 68.46, 13008-13015. Online: https:/ / bit.ly/3xIThQf.

Alvarado, Y., R. Ferrer y N. Florida (2020). «Evaluación ex post al proyecto productivo alternativo de Bella Bajo Monzón». En: Ciencia Unemi 13.34, 47-58. Online: https://bit.ly/3ubF2kM.

Antoine, J. y col. (2017). "Assessment of the potential health risks associated with the aluminium, arsenic, cadmium and lead content in selected fruits and vegetables grown in Jamaica». En: Toxicology Reports 4, 181-187. Online: https: / / bit. ly/3xIuPhS.

Antolinez, E. y col. (2020). «Estado actual de la cacaocultura: una revisión de sus principales limitantes». En: Ciencia y Agricultura 17.2, 1-11. Online: https://bit.ly/3eLlxch.

Arévalo, E. y col. (2017a). «Heavy metal accumulation in leaves and beans of cacao (Theobroma cacao L.) in major cacao growing regions in Pe- 
ru». En: Science of The Total Environment 605-606, 792-800. Online: https:/ / bit.ly/33fbPt3.

Arévalo, H. y col. (2017b). «Metales pesados en suelos, hojas y granos de zonas cacaoteras del Perú». En: International Symposium on Cocoa Research (ISCR) 2017, 1-6. Online: https: / / bit.ly / 33AeRbJ.

Argüello, D. y col. (2019). «Soil properties and agronomic factors affecting cadmium concentrations in cacao beans: A nationwide survey in Ecuador». En: Science of The Total Environment 649, 120-127. Online: https://bit.ly/2Re36Vs.

Arvelo, S. y col. (2017). Estado actual sobre la producción, el comercio y cultivo del cacao en américa. Online: https://bit.ly/3ecahXm. IICA-bba.

Barraza, F. y col. (2017). "Cadmium bioaccumulation and gastric bioaccessibility in cacao: A field study in areas impacted by oil activities in Ecuador». En: Environmental Pollution 229, 950-963. Online: https://bit.ly/3tDWPA2.

Barraza, F. y col. (2019). «Cadmium isotope fractionation in the soil cacao systems of Ecuador: a pilot field study». En: The Royal Society of Chemistry 9.58, 34011-34022. Online: https: / / rsc.li/ 3vBUS8x.

Bravo, I., C. Arboleda y F. Martin (2014). «Efecto de la calidad de la materia orgánica asociada con el uso y manejo de suelos en la retención de cadmio en sistemas altoandinos de Colombia». En: Acta Agronómica 63.2, 164-174. Online: https: / / bit.ly/3ePtXPD.

Casteblanco, J. (2018). «Heavy metals remediation with potential application in cocoa cultivation». En: La Granja 27.1, 21-35. Online: https://bit.ly/ 33ffMhn.

Celis, R., N. Florida y A. Rengifo (2020). «Impacto sobre indicadores físicos y químicos del suelo con manejo convencional de coca y cacao». En: CIENCIA UNEMI 13.33, 1-9. Online: https:/ / bit. ly/3vyOZJe.

Chávez, E. y col. (2015). «Concentration of cadmium in cacao beans and its relationship with soil cadmium in southern Ecuador». En: Science of The Total Environment 533, 205-214. Online: https: / / bit.ly/3eMSDIF.

Chocce, D. (2015). «Funciones de la Hoja de Coca durante el proceso de Violencia Política en el centro poblado de San José de Villa Vista, distrito de Chungui, provincia La Mar, región Ayacucho.» tesis de grado. Facultad de Antropología-
Universidad Nacional del Centro del Perú. Online: https:// bit.ly/3taqe4j.

Cunha, L. (2018). Estudo de competitividade do cacau e chocolate no Brasil: desafios na produção e comércio global. Ministério do Desenvolvimento, Indústria e Comércio Exterior-MDIC. Brasil. Online: https:/ / bit.ly/3gSHicM.

Díaz, U. y col. (2018). «Determinación de Cadmio y Plomo en almendras de cacao (Theobroma cacao), proveniente de fincas de productores orgánicos del cantón Vinces». En: Espirales 12.15, 77-92. Online: https:/ / bit.ly/3ebPZgv.

Engbersen, N. y col. (2019). "Cadmium accumulation and allocation in different cacao cultivars». En: Science of The Total Environment 678.678, 660-670. Online: https://bit.ly/3edGmOc.

Florida, N. y col. (2019). «Efecto de compost y NPK sobre los niveles de microorganismos y cadmio en suelo y almendra de cacao». En: Journal of High Andean Research 21.4, 264-273. Online: https:/ / bit.ly/3nLIHDm.

Florida, R., M. Claudio y B. Gómez (2018). «El pH y la absorción de cadmio en almendras de cacao orgánico (Theobroma cacao L.) en Leoncio Prado, Huánuco, Perú». En: Folia Amazónica 27.1, 1-8. Online: https://bit.ly/3nNNxjx.

Furcal, P. y J. Torres (2020). «Determinación de concentraciones de cadmio en plantaciones de Theobroma cacao L. en Costa Rica». En: Tecnología en Marcha 33.1, 122-137. Online: https: / / bit.ly/3eXdysN.

Gramlich, A. y col. (2017). «Cadmium uptake by cocoa trees in agroforestry and monoculture systems under conventional and organic management». En: Science of The Total Environment 580, 677-686. Online: https://bit.ly/3nXXJGp.

Gramlich, A. y col. (2018). «Soil cadmium uptake by cocoa in Honduras». En: Science of The Total Environment 612.580, 370-378. Online: https:/ / bit. ly/2SsNYEj.

Gunnar, N. (2013). «Encyclopaedia of health and safety at work». En: vol. 63. 10. Cap. Metals: chemical properties and toxicity. Online: https: / / bit.ly/3b0UCrP.

He, S. y col. (2015). «Soil biogeochemistry, plant physiology, and phytoremediation of cadmiumcontaminated soils». En: Advances in Agronomy 134, 135-225. Online: https:/ / bit.ly /3gZEIll.

Hernández, C. y col. (2017). «Metales pesados en suelos, hojas y granos de zonas cacaoteras del Perú». En: International Symposium on Cocoa Re- 
search (ISCR) 2017, 13-17. Online: https:/ / bit.ly / 3ok4tP3.

INEI (2017). Encuesta nacional Agropecuaria 2017 Principales resultados pequeñas, medianas y grandes unidades agropecuarias. Instituto Nacional de Estadística e Informática. Online: https: / / bit.ly / 3xZloe9.

Jiménez, C. (2015). «Global legal status of cadmium in cacao (Theobroma cacao): a fantasy or a reality». En: Producción+ Limpia 10.1, 89-104. Online: https:/ / bit.ly/2QTDltM.

Kabata, A. (2010). Trace elements in soils and plants. Taylor Francis Group. Online: https: / / bit.ly / 3uH0y0L.

Kruszewski, B., O. Wiesław y J. Kowalska (2018). «Nickel, cadmium and lead levels in raw cocoa and processed chocolate mass materials from three different manufacturers». En: Journal of Food Composition and Analysis 66, 127-135. Online: https://bit.ly/3ejjDAE.

Lanza, J. y col. (2016). «Evaluación del contenido de metales pesados en cacao (teobroma cacao 1.) de santa bárbara del Zulia, Venezuela». En: Saber 28.1, 106-115. Online: https://bit.ly/3h9OsJD.

López, Y., M. Cunias e Y. Carrasco (2020). «El cacao peruano y su impacto en la economía nacional». En: Revista Universidad y Sociedad 12.3, 344-352. Online: https:/ / bit.ly/3tqF9rj.

MINAGRI (2016). Estudio del cacao en el Perú y en el mundo; Situación Actual y Perspectivas en el Mercado Nacional e Internacional al 2015. MINAGRIDGPA-DEEIA. Online: https:/ / bit.ly/3bb7DyY.

- (2019). «Ministerio de Agricultura y RiegoDirección General de Políticas Agrarias; Dirección de Estudios Económicos e Información Agraria. Observatorio del Cacao». Online: https: //bit.ly/33sWhCl.

MINAGRICULTURA (2004). Ministerio de Agricultura y Desarrollo Rural. El beneficio y características fisicoquimicas del cacao (Theobroma cacao L.) Inf. téc. MINAGRICULTURA- FEDECACAO Colombia. Online: https:/ / bit.ly/3etUHX7.

Meter, A., R. Atkinson y B. Laliberte (2019). admio en el cacao de América Latina y el Caribe. Análisis de la investigación y soluciones potenciales para la mitigación. 84pp. Bioversity International. Online: https:/ / bit.ly/3baFnwA. Roma.

Mite, F., M. Carrillo y W. Durango (2010). «Avances del monitoreo de presencia de cadmio en almendras de cacao, suelos y aguas en Ecuador». En:
2010 Actas XII Congreso Ecuatoriano de la Ciencia del Suelo, 1-21. Online: https:/ /bit.ly/3uwZk81.

Morales, J., A. García y E. Méndez (2012). «¿Qué sabe usted acerca de... Cacao?» En: Revista Mexicana de Ciencias Farmacéuticas 43.4, 79-81. Online: https://bit.ly/3vHJBDI.

Oliveira, A. y col. (2019). «Cádmio e chumbo em amêndoa de cacau do Brasil». En: 2019 Anais do $13^{\circ}$ Simpósio Latino Americano de Ciência de Alimentos (SLACA), 1-2. Online: https: / / bit.ly / 3nXtoaM.

Pastor, S. (2017). «Niveles de cadmio en el chocolate: NM y ECA, sí; OTC, no». En: International Symposium on Cocoa Research (ISCR) 2017, págs. 1-5.

Pastor, S. y M. Gutierrez (2016). «Sobre Los Fundamentos De Una Norma Que Regula El Cadmio En Los Alimentos Y Presume Del Mismo En El Suelo». En: Memorias del XXI Congreso Latinoamericano de la Ciencia del Suelo, 24-28. Online: https: //bit.ly/3bbe4SE.

Pérez, G. y C. Azcona (2012). «The effects of cadmium on health». En: Magazine of Specialties Médico-Quirúrgicas 17.3, 199-205. Online: https: //bit.ly/3bbiSHA.

Prieto, J. y col. (2009). "Contaminación y fitotoxicidad en plantas por metales pesados provenientes de suelos y agua». En: Tropical and subtropical Agroecosystems 10.1, 29-44. Online: https: / / bit. ly/3o4Y7CP.

Raju, M. y col. (2020). «Cocoa-laden cadmium threatens human health and cacao economy: A critical view». En: Science of The Total Environment 720.10, 137645. Online: https:/ / bit.ly/3trMbfl.

Reyes, Y. y col. (2016). «Contaminación por metales pesados: Implicaciones en salud, ambiente y seguridad alimentaria». En: Ingeniería Investigación y Desarrollo 16.2, 66-77. Online: https: / / bit.ly / 3uEHhgD.

Romero, D. y col. (2019). «Content and the relationship between cadmium, nickel, and lead concentrations in Ecuadorian cocoa beans from nine provinces». En: Food control 106.106750, 1-8. Online: https:/ / bit.ly/3fdW9fd.

Sánchez, Á. y col. (2016). «Caracterización bromatológica de los productos derivados de cacao (Theobroma cacao L.) en la Chontalpa, Tabasco, México». En: Revista mexicana de ciencias agrícolas 7.1, 2817-2830. Online: https: / / bit.ly / 3eXHCnY.

Sánchez, N., C. Rivero e Y. Martínez (2011). «Cadmio disponible en dos suelos de Venezuela: efec- 
to del fósforo». En: Revista Ingeniería UC 18.2, 7-14. Online: https://bit.ly/3vHNYyC.

Tantalean, E. y M. Huauya (2017). «Distribución del contenido de cadmio en los diferentes órganos del cacao CCN-51 en suelo aluvial y residual en las localidades de Jacintillo y Ramal de Aspuzana». En: Revista de Investigación de Agroproducción Sustentable 1.2, 69-78. Online: https: / / bit.ly / $3 \mathrm{hb} 07 \mathrm{rN}$.

Unión Europea-UE (2014). Regulation No. 488/2014. They modify EC Regulation No 1881/2006 regarding the maximum content of cadmium in food products.
Inf. téc. Official Journal of the European Union. Online: https://bit.ly/3uvfOxT.

Viteri, O. (2013). «Evaluación de la Sostenibilidad de los cultivos café y cacao en las provincias Orellana y Sucumbíos-Ecuador». Tesis doctoral. Universidad Autónoma de Barcelona. Online: https://bit.ly/3nZY3o4.

Zug, K. y col. (2019). «Cadmium Accumulation in Peruvian Cacao (Theobroma cacao L.) and Opportunities for Mitigation». En: Water, Air, Soil Pollution 230.3, 72-80. Online: https: / / bit.ly / 3to8x1q. 